\title{
Open Science zwischen sozialen Strukturen und Wissenskulturen
}

Eine wissenschaftssoziologische Erweiterung

Werner Reichmann, Institut für Soziologie, Universität Konstanz, Fach 46, 78462 Konstanz (werner.reichmann@uni-konstanz.de)

Der vorliegende Beitrag plädiert für eine differenzierte Interpretation der Open-Science-Idee, nämlich sowohl als umfassendes strukturelles als auch als kulturelles Phänomen. In der öffentlichen Diskussion wird Open Science oftmals auf die strukturelle Öffnung des Publikationsmarktes für die Nachfrageseite reduziert. Dabei wird vernachlässigt, dass Wissenschaft auch aus darüberhinausgehenden Strukturen besteht, beispielsweise der Sozialstruktur wissenschaftlicher Gemeinden, bei denen Mechanismen der Schließung und Öffnung zu beobachten sind. Open Science sollte darüber hinaus als kulturelles Phänomen interpretiert werden. Unter Verwendung des Begriffs „Wissenskulturen“ zeigt der Beitrag, dass sich Open Science in der wissenschaftlichen Praxis als prozesshaftes und heterogenes Phänomen darstellt und dass Offenheit für verschiedene Gruppen der wissenschaftlichen Gemeinschaft unterschiedliche Bedeutungen aufweist.

\section{Open Science between social structures and epistemic cultures A Conceptual Complement from a Science Studies Perspective}

This paper argues for a differentiated understanding of Open Science. It suggests two interpretations: as a comprehensive structural and a cultural phenomenon. In public and political debates, Open Science is often reduced to strategies of opening up the structures of the publication market to ensure open access to scientific journal articles. Yet, there are other structures that may form the notion of Open Science, for example, the social structures of the scientific community, which are closed for certain social groups as recent data impressively shows. Furthermore, Open Science is a cultural phenomenon. Drawing on the notion of "epistemic cultures", this paper shows that Open Science is not a universal concept but is performed in quite different ways and has different meanings for different groups in the scientific community.

KEYWORDS: open science, social structure, social closure, epistemic cultures

This is an article distributed under the terms of the Creative Commons Attribution License CCBY 4.0 (https://creativecommons.org/licenses/by/4.0/)

https://doi.org/10.14512/tatup.26.1-2.43

Eingereicht: 14.03.2017. Angenommen: 18. 05.2017

\section{Einleitung}

Dieser Beitrag widmet sich der Frage, was der Begriff Open Science aus einer wissenschaftssoziologischen Perspektive heraus bedeuten und wie er durch eine solche Betrachtungsweise geschärft werden kann. Er macht deutlich, dass die aktuelle Verwendung des Begriffs in der öffentlichen Diskussion unnötig verengt ist.

In den letzten Jahren werden Wissenschaftlergemeinden aller Disziplinen zunehmend ermuntert, Wissenschaft offener zu gestalten. Als Schlagwort dient dabei der englische Begriff Open Science, und viele im weitesten Sinne wissenschaftspolitische Organisationen haben entsprechende Strategien erstellt, wie Wissenschaft zu öffnen sei. Dabei werden große Hoffnungen in Open Science gesetzt: Sie stelle eine neue Phase in der Wissenschaftsgeschichte dar, in der der freie Zugang und Austausch von wissenschaftlichem Wissen sichergestellt werde - auch für jene, die sich nicht hauptberuflich der Wissenschaft widmen, sich aber über aktuelle Forschung informieren und diese gegebenenfalls kommerziell nutzen wollen. Damit geht die gesellschaftspolitische Hoffnung einher, die Entfremdung zwischen Wissenschaft und Gesellschaft zu überwinden. Zudem sei die Idee der Open Science auch dazu geeignet, die öffentliche Finanzierung der Forschung stärker zu legitimieren (z. B. European Commission 2016; BMBF 2016; The Royal Society 2012; o. A. 2016). Allerdings gibt es auch Kritik an den Bestrebungen zur Öffnung der Wissenschaft. So wird beispielsweise argumentiert, dass sie den Einfluss kapitalistischer Logiken auf die Wissenschaften forciere (Hagner 2016) oder dass die verordnete Umstrukturierung von Publikationspraktiken einen Eingriff in die in Deutschland grundgesetzlich geschützte wissenschaftliche Freiheit darstelle (Reuß 2015).

Das Ziel dieses Beitrags ist es, den Open-Science-Begriff zu soziologisieren, indem zwei wissenschaftssoziologische Erweiterungen vorgeschlagen werden. Erstens soll das Konzept der strukturellen Offenheit von Wissenschaft, das in der oben skizzierten Debatte vor allem als strukturelle Öffnung wissen- 
schaftlicher Publikationsmärkte für die Nachfrageseite verstanden wird, erweitert werden. Sowohl wissenschaftliche Institutionen als auch Wissenschaftler selbst sind in wesentlich vielfältigere Strukturen eingebettet, als dies in der derzeitigen Debatte diskutiert wird, beispielsweise in Kollaborationsstrukturen, in Genderstrukturen, in Finanzierungsstrukturen, in soziale Strukturen etc. Auch diese Strukturen können jeweils offen oder geschlossen ausgestaltet sein, wobei es sich dabei nicht um eine einfache Dichotomie handelt, sondern um graduelle Öffnungen und Schließungen. Geschlossen sind Strukturen dann, wenn sie Barrieren aufbauen, die eine Teilhabe restriktiv steuern bzw. deren Öffnungen von „Gatekeepern“ (Crane 1967) überwacht werden, welche über die strukturelle Eingliederung entscheiden können. Strukturen sind dann offener, je weniger reguliert oder je gleicher Partizipationsmöglichkeiten verteilt sind. Eine solche Konzeptualisierung von Open Science als Struktur entspricht zwar dem Gebrauch des Begriffs, der in der öffentlichen Diskussion regelmäßig zur Anwendung gebracht wird. Hierbei kommt es allerdings zu einer unnötigen Verengung auf die Strukturen wissenschaftlicher Publikationsmärkte. Alle anderen Strukturen, die Wissenschaft ebenso schließen oder öffnen, werden in dieser Debatte nicht berücksichtigt. Die erste hier vorgeschlagene Ausweitung des Open-Science-Begriffs besteht daher darin, auch andere als lediglich die Strukturen des Publikationsmarktes zu fokussieren.

Zweitens soll der in der Wissenschaftssoziologie etablierte Kulturbegriff in die Diskussion eingebracht werden. Es wird argumentiert, dass Open Science auch als Wissenskultur gedacht werden kann, das heißt als praxisbezogenes Wissen darüber, „wie wir wissen, was wir wissen “ (Knorr Cetina 2002, S. 11, Herv. i. O.) und wie wissenschaftliches Wissen fixiert, bewertet in der Wissenschaftssoziologie etablierten Betrachtungsweisen auf Wissenschaft ergänzt und ausgeweitet. Beide Erweiterungen von Open Science, als vielfältige und umfassende Struktur und als Kultur des Wissens, werden im Folgenden theoretisiert und anhand aktueller empirischer Studien beispielhaft illustriert. Das Ziel ist es, in zwei Schritten zu zeigen, wie die wissenschaftssoziologische Formulierung eines umfassenden Open-Science-Begriffs gestaltet sein kann und dass Open Science nicht auf wenige Bedeutungsdimensionen von Marktstrukturen reduziert werden sollte.

\section{Open Science als Struktur}

Wenn Open Science in der gegenwärtigen Diskussion als strukturelles Phänomen begriffen wird, wird vor allem die Öffnung der Strukturen von Publikationsmärkten, in denen sich Wissenschaftler bewegen, thematisiert. Wissenschaftliche Publikationsmärkte sind politisch unter Druck geraten; die Kritik richtet sich dabei insbesondere gegen die Konzentration einiger weniger Verlage auf dem Markt wissenschaftlicher Zeitschriften. Diese Verlage haben große Marktmacht und können dementsprechend den Preis für die Zeitschriftenabonnements einseitig bestimmen. Dies führt dazu, dass ein großer Teil der Gesellschaft strukturell von der Möglichkeit ausgeschlossen wird, das in diesen Zeitschriften veröffentlichte wissenschaftliche Wissen rezipieren zu können.

Die Reduktion des Open-Science-Begriffs auf das Ziel, diese geschlossenen Strukturen des wissenschaftlichen Publikationsmarktes dahingehend $\mathrm{zu}$ verändern, dass wissenschaftliches Wissen allen zugänglich ist, ist irreführend. Die Idee von Open

\section{Die vorgeschlagene Ausweitung des Open-Science-Begriffs besteht darin, auch andere als lediglich die Strukturen des Publikationsmarktes zu fokussieren.}

und kommuniziert wird. Statt ausschließlich den institutionellen und strukturellen Aufbau und die (rechtlichen) Rahmenbedingungen von Wissenschaft zu analysieren, plädiert das Konzept der Wissenskulturen dafür, die Praxis der Wissenschaften und das ,science in the making“ (Latour 1987) in den Blick zu nehmen. Ein solches Verständnis von Open Science bekommt in der öffentlichen Diskussion kaum Aufmerksamkeit. Dabei zeigen aktuelle Studien, dass auch Wissenskulturen zu einer Öffnung oder Schließung von Wissenschaft beitragen können. Der zweite hier vorgeschlagene Schritt zur Erweiterung des OpenScience-Begriffs besteht daher in dem Plädoyer, den Blick nicht ausschließlich auf die Strukturen zu legen, sondern ihn auch hin zu Wissenskulturen zu wenden.

Das Verständnis von Open Science wird durch diese beiden
Science sollte nicht nur darauf fokussieren, wer wissenschaftliche Artikel lesen kann, sondern sollte beispielsweise auch berücksichtigen, wer in die Lage versetzt wird, diese zu produzieren. Die Strukturen der Wissenschaft formieren also nicht nur die Grenzen des Wissensflusses, sondern beispielsweise auch die der sozialen Zusammensetzung der wissenschaftlichen Gemeinde; auch ihre Sozialstruktur kann offen oder geschlossen sein. Open Science in dem hier vorgeschlagenen Verständnis stellt also nicht nur die Frage danach, wer auf wissenschaftliches Wissen zugreifen kann, sondern berücksichtigt auch, wer strukturell in der Lage ist, wissenschaftliches Wissen herzustellen und zu publizieren: Welche sozialen Gruppen sind strukturell von der Möglichkeit, wissenschaftliches Wissen herzustellen, ausgeschlossen? Open Science sollte damit auch als Sozi- 
alstruktur gedacht werden, die steuert, wer wissenschaftliches Wissen erzeugen kann. Ein Blick auf aktuelle Daten über die sozialstrukturelle Zusammensetzung der wissenschaftlichen Gemeinde in Deutschland lässt nur den Schluss zu, dass es sich um eine geschlossene Sozialform handelt. Im Folgenden wird ein aktueller empirischer Überblick über die Sozialstruktur der wissenschaftlichen Gemeinde auf zwei Polen der wissenschaftlichen Karriere gegeben: Studenten und Professoren.

Studenten sind die jüngste Gruppe der wissenschaftlichen Gemeinde und gleichzeitig sind sie die Wissenschaftler von morgen. Ihre Sozialstruktur setzt sich fort in der Zusammensetzung der Wissenschaftlerkohorten in den kommenden Jahrzehnten. Wird jeder mögliche Weg zu einem Universitätsstudium berücksichtigt, beginnen $23 \%$ der Kinder aus nichtakademischen belegen damit die Selektion von Professoren durch soziale Herkunft und die damit einhergehende soziale Schließung der wissenschaftlichen Gemeinde auf ihrer höchsten Karrierestufe.

Diese soziale Geschlossenheit ist allerdings nicht über alle Disziplinen gleich verteilt: Sie ist in den Rechts- und Lebenswissenschaften am stärksten ausgeprägt. Dort weisen $80 \%$ der Professoren die höchste soziale Herkunft auf. An zweiter Stelle stehen die MINT-Fächer ${ }^{2}$, gefolgt von den Geistes- und Sozialwissenschaften, die die höchsten Anteile von Professoren mit niedriger sozialer Herkunft haben (Möller 2013, S. 352; Graf 2016). ${ }^{3}$

An dieser Stelle wird der Mehrwert einer soziologischen Betrachtung des Open-Science-Begriffs als strukturelles Phänomen deutlich, da sie hervorhebt, dass neben Marktstrukturen

\section{Die Bedeutung von Open Science kann nicht darauf reduziert werden, die Chancen der Wissensrezeption offen zu gestalten.}

Haushalten, d. h. solchen, in denen kein Elternteil einen akademischen Abschluss hat, ein Hochschulstudium. Wenn mindestens ein Elternteil einen Hochschulabschluss vorweisen kann, ist die Wahrscheinlichkeit, ein Hochschulstudium zu beginnen 3,3-mal höher (Middendorff et al. 2013, S. 11). Diese ungleich verteilten Chancen manifestieren sich in der Zusammensetzung der Studentenschaft. Im Sommersemester 2012 - das sind die neuesten zur Verfügung stehenden Daten - kamen $50 \%$ aller Studenten aus einem akademischen Haushalt, womit sie im Vergleich zur Gesamtgesellschaft deutlich überrepräsentiert sind (Middendorff et al. 2013, S. 12). Der seit Jahren steigende Anteil von Studenten aus akademischen Haushalten pendelt sich in den letzten Jahren auf hohem Niveau ein - gleichzeitig nimmt der Anteil von Studenten aus den untersten Bildungsschichten stets ab (Middendorff et al. 2013, S. 13 f.). Auch die Wahrscheinlichkeit des Studienabbruchs hängt in hohem Maße von der Bildung der Eltern ab (Heublein et al. 2003, S. 46; Möller 2013, S. 342).

$\mathrm{Am}$ anderen Ende der wissenschaftlichen Karriereleiter, auf der Professorenebene, zeigt sich die soziale Schließung in der Wissenschaft noch deutlicher. Eine empirische Analyse des sozialen Hintergrunds von Professoren in Nordrhein-Westfalen ${ }^{1}$ vergleicht die Sozialstruktur der Professorenschaft im Jahr 2013 mit jener der Gesamtgesellschaft zum Zeitpunkt des Studiums dieser Professoren (Möller 2013). Sie zeigt, dass Professoren aus einer sozial privilegierten Gruppe kommen: So sind Kinder von Eltern, die eine hohe berufliche Stellung innehatten, in der heutigen Gruppe der Professoren deutlich überrepräsentiert. Die Daten

1 Die Studie umfasst die Daten von 1340 der rund 5100 Professoren (inkl. Emeriti und apl. Profs) an Universitäten in Nordrhein-Westfalen und ist damit nicht für ganz Deutschland statistisch repräsentativ. Auf Grund der Größe des Samples und der Mobilität, die Professoren im Laufe ihrer wissenschaftlichen Biografie aufweisen, ist allerdings nicht davon auszugehen, dass NRW ein Sonderfall ist. auch die Sozialstruktur der Wissenschaft zu deren Schließung beiträgt. Die aktuellen Daten über die soziale Zusammensetzung der deutschen wissenschaftlichen Gemeinde machen das Ausmaß dieser sozial bedingten Verzerrung der Möglichkeit, wissenschaftlich zu arbeiten, deutlich: Nicht nur auf dem in der öffentlichen Diskussion fokussierten Publikationsmarkt existieren verschlossene Zugänge, sondern es findet sich auch eine Hürde, die hier als sozialstrukturelle Paywall bezeichnet werden soll. Erst durch einen spezifischen Bildungshintergrund wird für viele der Zugang zur wissenschaftlichen Gemeinde möglich. Die Bedeutung von Open Science kann daher nicht darauf reduziert werden, die Chancen der Wissensrezeption offen zu gestalten, wie es in der öffentlichen Debatte zur Open Science überwiegend der Fall ist. Vielmehr sollte auch die Öffnung der Wissensproduktion Bestandteil einer Open Science sein.

\section{Open Science als Wissenskultur}

Neben der skizzierten Interpretation von Open Science als strukturelles Phänomen bietet sich, so der zweite Teil des hier vorgetragenen Arguments, eine zweite Perspektive an, die in den Kern der Wissenschaft vordringt. Open Science ist auch ein kultursoziologisches Phänomen, das auf der Ebene sozialer Handlungen und kultureller Praktiken angesiedelt ist und demzufolge als eine (neue) Wissenskultur (Knorr Cetina 1991, 2002) interpretiert werden kann.

2 MINT steht für die Fächer Mathematik, Informatik, Naturwissenschaften, Technikwissenschaften.

3 Die Darstellung der sozialen Schließung der wissenschaftlichen Gemeinde muss an dieser Stelle aus Platzgründen unvollständig bleiben. Auch für andere soziale Gruppen stellt sich Wissenschaft als geschlossenes Feld dar, insbesondere für Frauen (Möller 2013, S.355f.). 
Um zu zeigen, wie Open Science als kulturelles Phänomen begriffen werden kann, wird zunächst die Idee der Wissenskultur vorgestellt. Anschließend wird anhand einer aktuellen empirischen Studie von Levin und Leonelli (2017) illustriert, worin die wissenskulturellen Dilemmata einer Open Science bestehen können, um abschließend zu untersuchen, welche Konsequenzen die Diskussion von Open Science als Wissenskultur für ihr Verständnis haben kann.

Der Begriff der Wissenskulturen entstand in einer vergleichenden Laborstudie in den naturwissenschaftlichen Disziplinen der Hochenergiephysik und der Mikrobiologie (Knorr Cetina 2002). Laborstudien sind ein wissenschaftssoziologischer
Genau dieser Frage widmet sich eine aktuelle Laborstudie (Levin und Leonelli 2017), die eine empirisch fundierte Perspektive darauf erarbeitet, wie Open Science praktisch hergestellt und ausgeführt wird. Levin und Leonelli (2017) verwerfen ganz im Sinne des oben skizzierten Kulturbegriffs - die Annahme, dass Offenheit ein One-size-fits-all-Konzept ist. Die Autorinnen argumentieren vielmehr, dass ein universelles Verständnis von Offenheit wesentliche Fragen über die Formen, Implikationen und Ziele von Offenheit, wie sie sich im Forschungsalltag performativ darstellen, ignoriert (Levin und Leonelli 2017, S. 281). Offenheit im Allgemeinen und Open Science im Besonderen werden daher von den Autorinnen nicht als A-priori-Kon-

\section{Open Science bedeutet für unterschiedliche Forschergruppen}

\section{Unterschiedliches. Open Science muss nicht als universeller Begriff, sondern als heterogenes Konzept verstanden werden.}

Ansatz, nach dem Wissenschaft nicht lediglich als kognitives, rationales und ausschließlich von Ideen bevölkertes Handlungsfeld konzipiert wird, sondern auch als soziale Praxis, die von implizitem Wissen gesteuert und verstanden wird. Der konkrete wissenschaftliche Prozess, wie Wissenschaft in der täglichen Praxis getan wird, wird in Laborstudien durch empirische, auf die Methode der teilnehmenden Beobachtung gestützte Arbeit erhellt. Laborstudien grenzen sich damit von traditioneller Ideengeschichte und Wissenschaftstheorie ab, die vor allem die bereits verfertigten wissenschaftlichen Produkte analysieren. $\mathrm{Zu}$ dem indiziert der Kulturbegriff, dass es Wissenskulturen (im Plural) gibt, und wendet sich damit gegen die Einheit und Universalität der Wissenschaft. Nach dieser Lesart ist Wissenschaft ein ausdifferenziertes und kulturell geprägtes Handlungsfeld.

Wissenskulturen werden definiert als ,diejenigen Praktiken, Mechanismen und Prinzipien, die, gebunden durch Verwandtschaft, Notwendigkeit und historische Koinzidenz, in einem Wissensgebiet bestimmen, wie wir wissen, was wir wissen" (Knorr Cetina 2002, S. 11, Herv. i. O.). In diesem exemplarischen Zitat wird einerseits die Betonung des Wissensbegriffs deutlich. Wissenskulturen sind in Feldern zu finden, in denen es um die Suche nach Wahrheit sowie um Wissensproduktion und -prozessierung geht. Andererseits liegt der Definition ein spezifisch praxistheoretisches Verständnis von Kultur zugrunde, welches als ein verkörperter Wissensbestand verstanden wird, der ein spezifisches Tun evoziert. Kultur produziert und wird produziert durch Handlungen und das Wissen darüber, wie sie zu tun ist. Der Fokus auf die Performanz von Wissenschaft und auf die Frage, wie Wissenschaft getan wird, öffnet die Wissenschaft für eine ethnografische Untersuchung - und unter dem Blickwinkel des Open-Science-Begriffs stellt sich dabei eine neue Frage: Wie wird Geschlossenheit und Offenheit in der Wissenschaft auf der mikrosoziologischen Ebene dargestellt, getan und performt? zept interpretiert, sondern als etwas, das in der wissenschaftlichen Praxis hergestellt und damit konstruiert wird.

Levin und Leonellis Hauptargument ist, dass Open Science praxistheoretisch als permanenter Bewertungsmodus (mode of valuing) zu verstehen sei. Diesen Bewertungen liegt ein kultureller Wissensbestand zu Grunde - beispielsweise darüber, welche Arten von wissenschaftlichen Ergebnissen als wertvoll und publizierbar erachtet werden, welche Arbeiten als wichtig angesehen werden, was gute und was schlechte Wissenschaft ist, welche Publikationsformate wissenschaftliche Autorität versprechen etc. Diese differenten Bewertungsmodi machten unterschiedliche Aspekte des Forschungsprozesses systematisch unsichtbar und stellten andere in den Vordergrund.

In ihrer empirischen Arbeit stoßen Levin und Leonelli (2017, S. 283) auf eine Reihe wissenskultureller Offenheitsdilemmata, die vor allem die im wissenschaftlichen Alltag unhinterfragten Grundannahmen betreffen und sich aus dem Aufeinanderprallen gegensätzlicher Bewertungsmodi ergeben. Dies betrifft beispielsweise grundlegende Annahmen über die Planung und Evaluation wissenschaftlicher Arbeit und über den Nutzen einzelner Tätigkeiten im Rahmen des Forschungsprozesses.

Ein Beispiel aus der Studie kann ein solches Offenheitsdilemma illustrieren. In ihm geht es darum, wie speziell gezüchtete Versuchsmäuse und das Wissen darüber, wie diese gezüchtet werden, mit anderen geteilt werden kann (Levin und Leonelli 2017, S. 286 ff.). Die Forscherinnen sind mit der Frage konfrontiert, wie (in welcher Form), wann (an welchen Zeitpunkten des Forschungsprozesses) und was (welche Teile der Versuchsmäuse) mit anderen geteilt wird. Sie müssen eine Balance herstellen zwischen der wissenschaftlichen Reputation, die aus ihrer Fähigkeit, diese Mäuse züchten zu können, entsteht, und dem Ziel, sie als Ressource allgemein zugänglich zu machen. Letzteres ermöglicht anderen Forschern die Erarbeitung weiterer wis- 
senschaftlicher Ergebnisse, die das wissenschaftliche Wissen insgesamt erweitern könnten; es führt aber auch dazu, dass die wissenschaftliche Reputation möglicherweise anderen zugeordnet wird. Je nachdem, wann, wie und was allgemein zugänglich gemacht wird, kann dies negative Effekte haben: Gibt man - im Sinn einer Open Science - das Wissen über die Zucht der Mäuse zu einem frühen Zeitpunkt heraus, wirkt sich das positiv auf die Reputation der Laborleitung aus, aber es werden die Publikationsmöglichkeiten und damit die Karrierechancen von Doktoranden und technischem Personal gemindert. Gibt man es dagegen spät heraus, können weitere Erkenntnisse nicht oder nicht so schnell produziert werden. An diesem Beispiel wird auch die Verschränkung von wissenskulturellen Praktiken und der Ausgestaltung von Strukturen deutlich: Da, wie Levin und Leonelli zeigen, durch eine frühe, offene Weitergabe der Labormäuse die Aufstiegschancen wenig etablierter Wissenschaftler gemindert werden könnten, sind sowohl Macht- als auch Sozialstrukturen von der Wissenskultur betroffen.

Derartige wissenskulturelle Dilemmata werden von Levin und Leonelli auch anhand der offenen Verbreitung von Software und von der Verfügbarmachung von quantitativen Daten im Detail analysiert. Auch in diesen Fällen stehen einander unterschiedliche Bewertungsmodi gegenüber, die gegeneinander abgewogen werden müssen und die die Form von Open Science kulturell prägen. Anhand dieser Beispiele wird zudem deutlich, dass Open Science für unterschiedliche Forschergruppen Unterschiedliches bedeutet. Open Science müsse demzufolge auf der empirischen Ebene nicht als universeller Begriff, sondern als heterogenes Konzept verstanden werden (Levin und Leonelli 2017, S. 288-289). Levin und Leonelli (2017) zeigen mit ihrer einer offenen Wissenschaft vernachlässigt werden: Meist wird die Forderung nach Open Science auf die Öffnung eines Teils des wissenschaftlichen Publikationsmarktes reduziert, seltener geht es auch um Software- und Datenmärkte. Andere strukturelle Seiten einer Open Science, wie beispielsweise die Sozialstruktur der wissenschaftlichen Gemeinde, werden hingegen ausgeblendet. Dabei zeigen aktuelle Daten, dass auch die sozialen Strukturen der Wissenschaft von sozialen Schließungsmechanismen betroffen sind. Der zweite blinde Fleck in der Open-Science-Diskussion besteht in der Vernachlässigung der Behandlung von Open Science als kulturellem und praxistheoretischem Phänomen. Open Science besteht aber ebenso wie aus spezifischen Strukturen aus einer spezifischen Wissenskultur, die sich in einem System aus wissenschaftlichen Praktiken und performativen Darstellungen im wissenschaftlichen Alltag niederschlägt. Sowohl die strukturelle als auch die kulturelle Betrachtungsweise zeigt, dass der Open-Science-Begriff aktuell zu eng geführt wird.

Die eingangs geforderte Soziologisierung des Open-ScienceBegriffs bedeutet hier also in einem ersten Schritt eine De-Ökonomisierung, da der Fokus weg von Marktstrukturen hin zu anderen, aus Sicht der Wissenschaftssoziologie ebenso wichtigen strukturellen Phänomenen gelenkt wird. Auf die sozialstrukturellen Schließungsmechanismen in der Wissenschaftlergemeinde Deutschlands wurde bereits empirisch eingegangen. Ähnliche Schließungstendenzen könnten auch in den wissenschaftlichen Kollaborationsstrukturen zu finden sein (beispielsweise Moody 2004) oder in den Machtstrukturen der Wissenschaft (beispielsweise Bourdieu 1975), die ebenso zu ihrer Schließung oder Öffnung beitragen können. Im zweiten Schritt bedeutet Soziologi-

\section{Open Science besteht aus einer spezifischen Wissenskultur, die sich in einem System aus wissenschaftlichen Praktiken und Performanzen im wissenschaftlichen Alltag niederschlägt.}

instruktiven Arbeit, dass Offenheit in den Wissenschaften nicht nur auf der strukturellen Ebene beobachtet werden kann, sondern dass auch lokal verankerte, wissenskulturell geprägte Formen der Öffnung von Wissenschaft existieren. Die Debatte um Open Science wird auf diese Weise um eine wissenskulturelle, eine soziale und, in manchen Fällen, sogar um eine moralische Dimension erweitert (Levin und Leonelli 2017, S. 284).

\section{Die Vielfalt von Open Science}

Basierend auf der analytischen Unterscheidung zwischen den Strukturen und Kulturen einer offenen Wissenschaft zeigt dieser Beitrag, dass in der derzeitigen öffentlichen und politischen Diskussion über Open Science wichtige inhaltliche Dimensionen sierung des Open-Science-Begriffs eine Kulturalisierung. Dabei wird ein spezifisches Verständnis von Kultur als kultureller Praxis (Knorr Cetina 1995, S. 128) verwendet, das Kultur als eine geteilte Ordnung an Bedeutungen, Symbolen und Wissen, das wissenschaftliches Handeln anleitet, ermöglicht und beschränkt (Reckwitz 2006, S. 84-85), bezeichnet. Für eine offene Wissenschaft bedeutet eine derartige konzeptuelle Kulturalisierung des Wissens, dass sich die Öffnung in den durch den gemeinsamen Wissensvorrat gesteuerten Praktiken niederschlägt.

Die Konzeption von Open Science als Wissenskultur hat als Konsequenz, dass die Öffnung von Wissenschaft nicht als in sich homogen und einheitlich ausgestaltet begriffen wird, sondern dass auch Open Science stets lokal verankert und sozial differenziert ist. Die Erkenntnis, dass Wissenschaft in ihrer Universalität limitiert ist, mag in der Wissenschaftssoziologie nicht 
neu sein (Galison und Stump 1996; Knorr Cetina 2002). Allerdings findet sie in der Diskussion um die Ausgestaltung einer Open Science bislang keine Berücksichtigung. Levin und Leonelli (2017) zeigen in ihrer empirischen Studie, dass auch Open Science kein universaler Begriff ist, woraus folgt, dass er weder in allen wissenschaftlichen Feldern gleich ausgestaltet sein sollte noch eine identische Anwendung finden kann. Die Debatte um Open Science sollte daher berücksichtigen, dass Definitionen von guter und schlechter Wissenschaft, von erstrebenswerten Ergebnisformen und davon, welche Arbeiten im wissenschaftlichen Prozess mehr oder weniger geschätzt werden, über Disziplinen ebenso wie über zeitliche und räumliche Kontexte variieren (Haeussler et al. 2009) und sich damit sowohl die strukturellen als auch die kulturellen Kontextbedingungen unterscheiden.

\section{Literatur}

BMBF (2016): Open Access in Deutschland. Die Strategie des Bundesministeriums für Bildung und Forschung. Online verfügbar unter https://www.bmbf.de/ pub/Open_Access_in_Deutschland.pdf, zuletzt geprüft am 12.06.2017. Bourdieu, Pierre (1975): The Specivity of the Scientific Field and the Social Conditions of the Progress of Reason. In: Social Science Information 14 (6), S. 19-47.

Crane, Diana (1967): The Gatekeepers of Science: Some Factors Affecting the Selection of Articles for Scientific Journals. In: The American Sociologist 2 (4), S. 195-201.

European Commission (2016): Open Innovation, Open Science, Open to the World - a Vision for Europe. Online verfügbar unter http://ec.europa.eu/ newsroom/dae/document.cfm?doc_id=16022, zuletzt geprüft am 12.06.2017.

Galison, Peter; Stump, David (Hg.) (1996): The Disunity of Science: Boundaries,

Contexts, and Power. Stanford: Stanford University Press.

Graf, Angela (2016): Eliten im wissenschaftlichen Feld Deutschlands - Sozialprofil und Werdegänge. In: Soziale Welt 67 (1), S.23-42.

Haeussler, Carolin; Jiang, Lin; Thursby, Jerry; Thursby, Marie C. (2009): Specific and General Information Sharing Among Academic Scientists. In: NBER Working Papers Series 15315. Online verfügbar unter http://www.nber.org/ papers/w15315, zuletzt geprüft am 12.06.2017.

Hagner, Michael (2016): \#0pen_Access: Wie der akademische Kapitalismus die Wissenschaften verändert (Geschichte der Gegenwart). Online verfügbar unter http://geschichtedergegenwart.ch/open_access-wie-derakademische-kapitalismus-die-wissenschaften-veraendert, zuletzt geprüft am 12.06.2017.

Heublein, Ulrich; Spangenberg, Heike; Sommer, Dieter (2003): Ursachen des Studienabbruchs. Analyse 2002. Hannover: HIS (HIS Hochschulplanung, Bd. 163).

Knorr Cetina, Karin (1991): Epistemic Cultures: Forms of Reason in Science. In: History of Political Economy 23 (1), S. 105-122.

Knorr Cetina, Karin (1995): Laborstudien - Der kultursoziologische Ansatz in der Wissenschaftsforschung. In: Renate Martinsen (Hg.): Das Auge der Wissenschaft - Zur Emergenz von Realität. Baden-Baden: Nomos, S. 101-135.

Knorr Cetina, Karin (2002): Wissenskulturen - Ein Vergleich naturwissenschaftlicher Wissensformen. Frankfurt am Main: Suhrkamp.

Latour, Bruno (1987): Science in Action - How to Follow Scientists and Engineers through Society. Cambridge, MA: Harvard University Press.
Levin, Nadine; Leonelli, Sabina (2017): How Does One „Open“ Science? Questions of Value in Biological Research. In: Science, Technology \& Human Values 42 (2), S. 280-305.

Middendorff, Elke; Apolinarski, Beate; Poskowsky, Jonas; Kandulla, Maren; Netz, Nicolai (2013): Die wirtschaftliche und soziale Lage der Studierenden in Deutschland 2012.20. Sozialerhebung des Deutschen Studentenwerks, durchgeführt durch das HIS-Institut für Hochschulforschung. Berlin: Deutsches Studentenwerk.

Möller, Christina (2013): Wie offen ist die Universitätsprofessur für soziale Aufsteigerinnen und Aufsteiger? Explorative Analysen zur sozialen Herkunft der Professorinnen und Professoren an den nordrhein-westfälischen Universitäten. In: Soziale Welt 64 (4), S. 341-360.

Moody, James (2004): The Structure of Social Science Collaboration Network: Disciplinary Cohesion from 1963 to 1999. In: American Sociological Review 69 (2), S. 213-238.

०. A. (2016): Amsterdam Call for Action on Open Science. Online verfügbar unter https://english.eu2016.nl/binaries/eu2016-en/documents/reports/ 2016/04/04/amsterdam-call-for-action-on-open-science/amsterdam-callfor-action-on-open-science.pdf, zuletzt geprüft am 12.06.2017.

Reckwitz, Andreas (2006): Die Transformation der Kulturtheorien - Zur Entwicklung eines Theorieprogramms. Weilerswist: Velbrück Wissenschaft.

Reuß, Roland (2015): Open Access: Der Geist gehört dem Staat. In: Frankfurter Allgemeine Zeitung, 30.12.2015. Online verfügbar unter http://www.faz.net/ aktuell/feuilleton/forschung-und-lehre/baden-wuerttemberg-entrechtetseine-wissenschaftlichen-autoren-13988149.html, zuletzt geprüft am 12.06.2017.

The Royal Society (2012): Science as an Open Enterprise. Online verfügbar unter https://royalsociety.org/ /media/Royal_Society_Content/policy/projects/ sape/2012-06-20-SAOE.pdf, zuletzt geprüft am 12.06.2017.

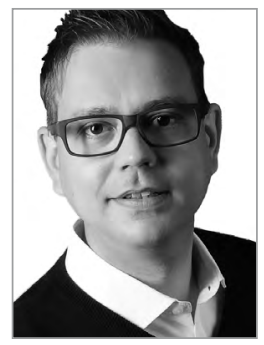

PD DR. WERNER REICHMANN

ist Privatdozent und wissenschaftlicher Mitarbeiter am Institut für Soziologie der Universität Konstanz. Forschungsschwerpunkte: Wissenschaftssoziologie, STS, Mediensoziologie, Interaktionssoziologie. 